\title{
STUDY OF BLUE PHOTOLUMINESCENT BAND IN ZnS CRYSTALS
}

\author{
N.Q. Liem, V.X. Quang and D.X. Thanh \\ Institute of Material Science, Nghia do-Tuliem, Hanoi, Vietnam \\ (Received November 9, 1993; revised version April 28, 1994; \\ final form September 1, 1994)
}

The zinc sulphide crystals as-grown and heat treated in different atmospheres, have been studied by photoluminescence technique. A blue emission band (IB) peaking at $2.91 \mathrm{eV}$ has been identified in the mentioned crystals. Parameters of the IB band such as peak energy, half width of spectrum, lifetime of photoluminescence as well as a superlinearly excitation intensity dependence of the luminescence have been determined. A model of the IB transition is proposed to explain the properties and features of IB luminescence.

PACS numbers: $78.55 .-\mathrm{m}, 78.55$.Et

\section{Introduction}

Zinc sulphide is a direct bandgap semiconductor with $E_{\mathrm{g}}=3.75 \mathrm{eV}$ at room temperature and exhibits very efficient luminescent transitions. The emission property of the crystals as well as of the powder has been studied by many researchers in the whole spectral range from the near band edge $[1,2]$ to the infrared region. In the visible region there is a very well-known emission band peaking at around $2.7 \mathrm{eV}$ being the so-called SA (self-activated) band. The nature of SA centre has been studied by numerous authors and it is concluded to be donor-acceptor (D-A) transition $[3,4]$. Another explanation for the appearance of a new emission band at the high energy side of the usual SA band is the transition within the SA. centre $[5,6]$.

During our study by photoluminescence (PL) spectroscopy on the ion implanted $\mathrm{ZnS}: \mathrm{Al}$ crystals we discovered the luminescent band related to the lattice damage [7]. This band was firstly named IB as "implantation blue" and has a maximum at around $2.87 \mathrm{eV}$. The nature of the IB transition is still not clarified but it was confirmed that $\mathrm{Zn}$-vacancy plays a very important role in this configuration. The experiments for Zn-vacancy concentration dependence of the IB emission intensity were done and also showed that Zn-vacancy could be created by annealing treatment [7]. In this paper we research several detailed features of the IB band, which came from thermally-induced $\mathrm{Zn}$-vacancies. A special property of the IB band is an excitation intensity dependence of PL intensity being superlinear. This will be discussed later. 


\section{Experimental procedures}

The samples for investigation were cut from an undoped bulk $\mathrm{ZnS}$ single crystal, which was grown by a melting method. Some of them were heat treated in molten zinc for 74 hours at $900^{\circ} \mathrm{C}$ (solvent extraction purification). Some of others were annealed at $400^{\circ} \mathrm{C}$ under saturated $\mathrm{Zn}$ vapour.

The techniques were used as follows:

- Photoluminescence excited by $365 \mathrm{~nm}$ and $333 \mathrm{~nm}$ lines of $100 \mathrm{~W}$ mercury high pressure lamp and $100 \mathrm{~W}$ xenon high pressure lamp for excitation spectra (herein we call it dc-PL).

- Time resolved PL with nitrogen laser pulse excitation and sampling 1S1 Tektronix. Nitrogen laser pulse has a width of $1 \mathrm{~ns}$, wavelength $337.1 \mathrm{~nm}$ and repetition rate $1-100 \mathrm{~Hz}$. Time resolution of $1 \mathrm{~S} 1$ sampling is $350 \mathrm{ps}$.

- Luminescence intensity is dependent on excitation intensities at different moments after exciting. By varying the polarized voltage to photomultiplier and using calibrated neutral glass filters for attenuating excitation beam, one could measure the luminescent intensity changing by four orders of magnitude on the excitation intensity.

- In our opinion, it is difficult to get exactly each proper band from a common spectrum, which is overlapped by some components. This work needs a good physics understanding on research samples. But several techniques for signal acquisition were used such as time-resolved spectroscopy, selected excitation, etc. On the other hand, computation by the Gauss-Newton for curve fitting and for separation of the spectrum into the components was performed. Our computation based on a suggestion that each component spectrum has the Gaussian shape.

- PL measurements were performed at the sample temperatures varying from $300 \mathrm{~K}$ down to $77 \mathrm{~K}$.

\section{Experimental results and discussions}

Figure 1 shows PL of samples at room and liquid nitrogen temperatures. Using the computation technique mentioned above we could show that it reveals a predominant emission band peaking at around $2.9 \mathrm{eV}$. The SA component occupied only a small range. Figure 2 shows dc-PL spectra when the samples are excited by $365 \mathrm{~nm}$ and $333 \mathrm{~nm}$ lines of mercury high pressure lamp. In this case a power drop onto the sample surface was estimated at about $10 \mathrm{~mW} / \mathrm{cm}^{2}$. With $365 \mathrm{~nm}$ excitation, which is the characteristic excitation of the SA transition [3], therefore only SA peak was observed. Basing on the characteristics of the PL, excitation spectrum with $333 \mathrm{~nm}$ characteristic excitation band, and a quenching in Zn saturated vapour annealing, it is confirmed that the $2.9 \mathrm{eV}$ band and IB band are identified [7].

Both types of samples, with and without solvent extraction purification, exhibit the same PL and excitation spectra. This means that the as-grown $\mathrm{ZnS}$ crystals are pure enough, or at least some impurities such as $\mathrm{Cu}, \mathrm{Ag}$, etc., which came unintentionally into the crystals during growth time play a minor role in the IB emission band. 


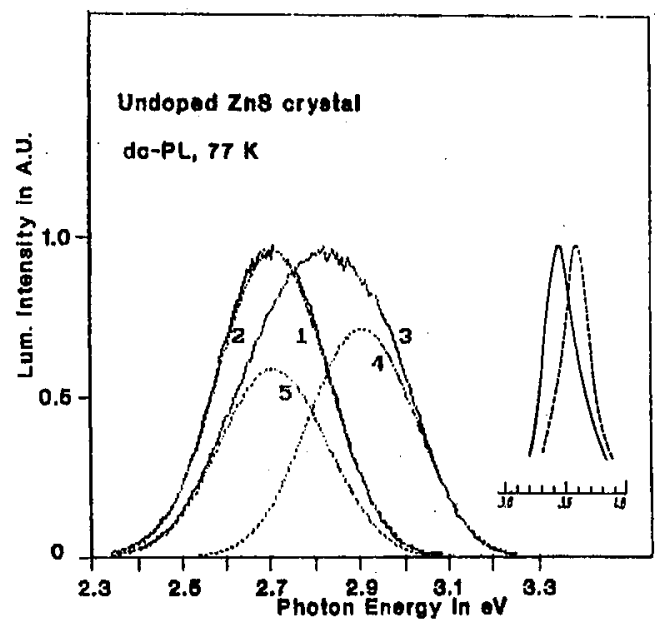

Fig. 1. PL of undoped $\mathrm{ZnS}$ crystal with nitrogen laser excitation at $77 \mathrm{~K}(1)$ and Gaussian fitting for the IB component (2), at $300 \mathrm{~K}$ (3) and Gaussian fitting for the IB (4) and SA (5) bands. Inset in the right corner is PL excitation spectra for SA (solid line) and IB (dashed line) at $77 \mathrm{~K}$.

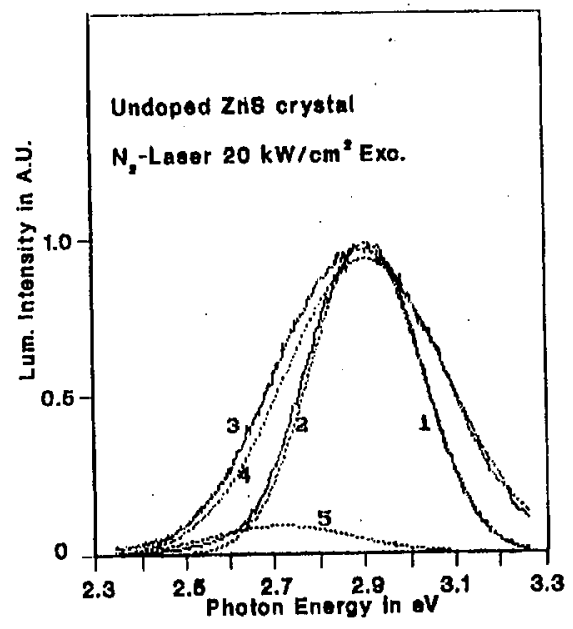

Fig. 2. dc-PL of undoped $\mathrm{ZnS}$ crystal at $77 \mathrm{~K}$ with $365 \mathrm{~nm}$ excitation (1) and Gaussian fitting for the SA band (2) with $333 \mathrm{~nm}$ line excitation (3) and Gaussian fitting for the IB (4) and SA (5) components.

In an attempt to understand the nature of the IB centre, measurements of the shifts of PL spectra following the excitation intensity, delay time excitation, sample temperature changes (from now on they are the so-called $J$-shift, $t$-shift, $T$-shift, respectively) and temperature dependence of half-width of the spectra 
were done. Lifetimes of the IB luminescence at $77 \mathrm{~K}$ and $300 \mathrm{~K}$ were also determined as $400 \mathrm{~ns}$ and $150 \mathrm{~ns}$, respectively. These values are about 10 times (at $77 \mathrm{~K}$ ) and about 17 times (at $300 \mathrm{~K}$ ) shorter than those of the SA band. The rapid decay at room temperature explains why in most dc-PL spectra the IB was not clearly visible beside the SA band. Of course, there are some other reasons why the IB band is not apparent in ZnS crystals but the strongest factor may be the excitation intensity dependence of this emission band intensity. The special feature of the IB band is that the excitation intensity dependence of PL intensity is superlinear as described below. The $J$-shift was measured at a fixed moment just after excitation by nitrogen laser pulse. This means that SA band would have the smallest superposition to the IB signal. In other words, the intensity ratios of IB signal over SA signal are the largest. The result of $J$-shift is that the emission peak moved only slightly to the lower energy side corresponding to the lower intensity excitation, while the common half-width was expanded. This shift is much smaller than the $J$-shift of the SA spectrum [8]. Computations on many sets of 8 spectra corresponding to different excitation rates from $200 \mathrm{~kW} / \mathrm{cm}^{2}$ down to $20 \mathrm{~W} / \mathrm{cm}^{2}$ were performed in order to subtract the SA component. In all above spectra intensity of the SA band varied almost linearly on excitation intensity change and occupied only a few percent in the normalized spectra (see Fig. 1 typically). Final results after curve fitting showed that the IB peak does not move at all. The slight $J$-shift shown above is caused by superposition of the SA and IB bands. We revealed the unique feature of the IB band, i.e. that the luminescence intensity depends superlinearly on the excitation intensity. Figure 3 shows curves based on the following formula:

$$
I=A L^{k},
$$

where $I$ is emission intensity, $L$ is excitation intensity and $A$ is the proportional coefficient. The values of $k=1.67$ and 1.5 were received at $300 \mathrm{~K}$ and $77 \mathrm{~K}$, respectively. It can be seen that intensity quenching at room temperature is stronger than that at lower temperatures. This factor explains why at ronm temperature and $333 \mathrm{~nm}$ line of mercury lamp excitation the IB band could not be observed (see also [7]). The time-resolved spectra were recorded at different delay times 0 , $30,50,100,150,200,300,400,600,1000,1500$ and $2000 \mathrm{~ns}$ from excitation laser pulse and at sample temperatures $77 \mathrm{~K}$ and $300 \mathrm{~K}$. The results of this measurement were similar to those of the $J$-shift experiment, e.g. a small movement of the common peak was due to the superposition of the SA band on the decayed IB luminescence. For much longer delays, only the SA band could be observed and it shifted following delay times as a consequence of $\mathrm{D}-\mathrm{A}$ transitions. Curve fitting based on the Gauss-Newton method revealed that there is no $t$-shift of the IB band. The $T$-shift was measured at different temperatures from $77 \mathrm{~K}$ to $300 \mathrm{~K}$. Luminescence occurred at a fixed moment just after excitation by nitrogen laser pulse. There is no $T$-shift of the IB band, either. The parameters and properties of the IB band are collected in Table. $E_{\mathrm{p}}$ is the energy peak and $W$ is the half-width of the IB band; $\tau$ is the lifetime of the IB transition. The shifts of spectra by excitation intensity $(J)$ or delay times $(t)$ or sample temperature $(T)$ changes have a usual meaning as showed above and as in other publications. 


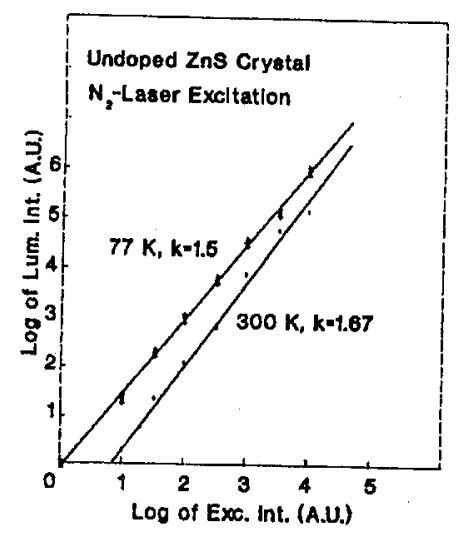

Fig. 3. The luminescence intensity dependence on the excitation intensity of an undoped $\mathrm{ZnS}$ crystal at $77 \mathrm{~K}$ and $300 \mathrm{~K}$.

TABLE

The parameters and features of the IB band in $\mathrm{ZnS}$ crystal at different temperatures.

\begin{tabular}{l|c|c|c|c}
\hline \hline & $300 \mathrm{~K}$ & $200 \mathrm{~K}$ & $130 \mathrm{~K}$ & $77 \mathrm{~K}$ \\
\hline$E_{\mathrm{p}}[\mathrm{eV}]$ & 2.91 & 2.91 & 2.91 & 2.91 \\
$W[\mathrm{meV}]$ & 400 & 380 & 360 & 290 \\
$\tau[\mathrm{ns}]$ & 150 & - & - & 400 \\
$J$-shift & no & - & - & no \\
$t$-shift & no & - & - & no \\
$T$-shift & no & no & no & no \\
$k$ & 1.67 & - & - & 1.5
\end{tabular}

From the obtained results we can confirm that the nature of IB transition is unlikely $\mathrm{D}-\mathrm{A}$ recombination. In the past, some authors wrote about the existence of a fast decay component with a time constant about $400 \mathrm{~ns}$ at $77 \mathrm{~K}$ when measuring the decay characteristics of the SA band [5]. In Ref. [9] the authors recorded a luminescence band peaking at around $425 \mathrm{~nm}$ in undoped $\mathrm{ZnS}$ crystals. The $425 \mathrm{~nm}$ band of undoped $\mathrm{ZnS}$ shows a shift neither with increasing delay time nor with decreasing temperature and may be attributed to the transition within the localized centre associated with some defects. In our opinion, in each of the papers $[5,7,9]$ the authors failed to show conclusively the characteristics of the blue emission band, which we identified as the IB band.

Recently, some publications have reported a superlinear excitation power dependence of the near band edge photoluminescence of CdTe compound [10, 11]. At sample temperatures low enough for neglecting thermal dissociation of free and bound excitons and with the assumption that the electron concentration in 
the conduction band is equal to the concentration of holes in the valence band, the coupled differential equations have been deduced to explain the experimental results. The authors stated that their theory can be applied to other II-VI or III-V semiconductors. In this case, at relatively high sample temperatures it is possible to omit some terms belonging to the excitonic processes. The illustration of the IB transition can be seen in Fig. 4. The numbers marked the ordinal transitions to emit IB photons. Briefly, the transitions can be as follows:

1. The first process (number 1 in Fig. 4) causes absorption and creates electrons in the conduction band with the same number of holes in valence band.

2. Secondly, the electrons from (Zn-vacancy $+\ldots$ ) complex, responsible for the IB centre, should fall down to the valence band (marked by number 2). One part of conduction electrons should also relax to some certain donors (marked by number $2^{\prime}$ ).

3 . The third process (number 3 ) is a recombination of the electrons and holes from the conduction band and (Zn-vacancy $+\ldots$ ) complex, respectively.

With the presented model, depending on the concentrations of donors and acceptors in the crystals, the superlinear excitation intensity dependence of the IB luminescence could be explained.

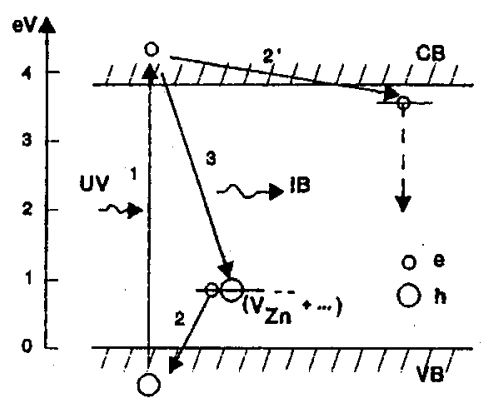

Fig. 4. Model might be proposed for the IB transition. The detailed explanations are in the text.

\section{Conclusions}

The blue emission band named IB band of the undoped zinc sulphide crystals has been studied relatively in detail. The parameters of peak energy, luminescence lifetime, as well as spectral half-width at different sample temperatures have been reported. The characteristics and properties such as no $J$-shift, no $t$-shift, etc. and the superlinear excitation intensity dependence of the IB luminescence have been observed. The model of the IB centre is proposed to be a conduction band-acceptor. This acceptor consists of Zn-vacancy and an unidentified part, which is involved in other donors and acceptors. It can be confirmed that the IB centre has a native nature in the $\mathrm{ZnS}$ crystal and "IB" is the historic name only, because we revealed this emission band for the implanted samples for the first 
time. The investigations of electronic structure and detailed configuration of the IB centre must be continued.

\section{Acknowledgments}

The author wishes to thank Dr. P.H. Duong for manuscript preparation, Mr. N.V. Quang and Mr. N.V. Manh for technical service.

\section{References}

[1] T. Taguchi, T. Yokogawa, H. Yamashita, Solid State Commun. 49, 551 (1984).

[2] J. Gutowski, I. Broser, G. Kudlek, Phys. Rev. B 39, 3670 (1989).

[3] K. Era, S. Shionoya, Y. Washizawa, J. Phys. Chem. Solids 29, 1827 (1968); K. Era, S. Shinoya, Y. Washizawa, H. Ohmatsu, J. Phys. Chem. Solids 29, 1843 (1968).

[4] J.R. James, B.C. Cavenett, J.E. Nicholls, J.J. Davies, D.J. Dunstan, J. Lumin. 12/13, 447 (1976).

[5] T. Koda, S. Shionoya, Phys. Rev. 136, A541 (1964).

[6] S. Oda, H. Kukimoto, J. Lumin. 18/19, 829 (1979).

[7] V.X. Quang, T.K. Anh, N.Q. Liem, K. Mass, B. Selle, Phys. Status Solidi A 79, K181 (1983).

[8] A. Abdel-Kader, H.I. Farag, J. Mater. Sci. 23, 2382 (1988).

[9] J. Zhou, H. Goto, N. Sawaki, I. Akasaki, J. Lumin. 35, 255 (1986).

[10] D.E. Cooper, J. Shirafuji, P.R. Newmann, J. Cryst. Growth 86, 544 (1988).

[11] T. Schmidt, G. Daniel, K. Lischka, J. Cryst. Growth 117, 748 (1992). 